\title{
Dynamic structure factor of a dilute Lennard-Jones gas
}

\author{
Kunimasa Miyazaki* and I. M. de Schepper \\ IRI, Delft University of Technology, 2629 JB Delft, The Netherlands \\ (Received 31 January 2001; published 11 May 2001)
}

\begin{abstract}
We calculate the leading correction term in the density expansion of the dynamic structure factor for a Lennard-Jones fluid using the Boltzmann equation. The qualitative behavior is found to be very similar to the hard-sphere result reported by Kamgar-Parsi et al. [Phys. Rev. A 35, 4781 (1987)]. A comparison was made with the results from the neutron scattering experiment by Verkerk et al. [Phys. Rev. Lett. 67, 1262 (1991)] for dilute argon gases. Several possibilities to explain the discrepancies of the present results from the experiment are proposed.
\end{abstract}

DOI: 10.1103/PhysRevE.63.060201

PACS number(s): 51.10.+y, 61.20.Ne

\section{INTRODUCTION}

The density-expansion approach is an exact and therefore a very useful way to study intermolecular potentials of dilute gases. Virial expansions of the equation of state are commonly used for this purpose [1]. Recent developments of experimental techniques paved the way to investigate this approach in a more microscopic and direct way. The neutron-diffraction method, for example, was used to measure the static structure factor $S(k)$ for rare gases [2-4]. The Fourier transformation of the leading order of the density expansion of $S(k)$ yields the potential directly. The dynamic structure factor $S(k, \omega)$ is another quantity which would provide the useful information of the intermolecular potentials, because dynamical properties of dilute gases would be as sensitive to the shape of the potential as the static properties. A decade ago, Verkerk et al. conducted inelastic neutron scattering experiments and observed the linear term in the density expansion of $S(k, \omega)$ for dilute argon for the first time [5]. They compared their results with the theoretical calculation for the hard-sphere fluid obtained using the Boltzmann equation [6]. It was found that, for the small wave vector $\left(k=0.3 \mathrm{~nm}^{-1}\right)$, the experimental result agrees qualitatively with the hard-sphere theory but for larger wave vectors $\left(k \geqslant 0.7 \mathrm{~nm}^{-1}\right)$, the discrepancies are pronounced, especially at small frequencies. The theoretical calculation predicts that the dynamic structure factor is scaled by a reduced frequency and, if plotted against this reduced frequency, it should be independent of the wave vector. Their experimental results, however, showed very sensitive dependence on the wave vector. In order to explain the discrepancies, they drew two conjectures from their observations: (i) Sensitivity of $S(k, \omega)$ to the shape of the intermolecular potential is the origin of the discrepancies between the hardsphere theory and experiment. Since only the binary collision of a pair of atoms contributes to the linear term of $S(k, \omega)$ and the observations were made for shorter length scale than the mean free path, the result would reflect the detail of a single collision process and, therefore, might be very sensitive to the shape of the potential. (ii) The thermodynamic parameters used in their experiments might not have satisfied

*Electronic address: k.miyazaki@iri.tudelft.nl the criteria of the density expansion. In order for the density expansion of the dynamic structure factor to be valid, either wave vector or frequency, which are scaled by mean free path and mean free time, respectively, must be large. The size of both of them was critical at the region where the discrepancies from the theoretical prediction were large.

In this Rapid Communication, we concentrate on the first conjecture and calculate the leading term of the density expansion of $S(k, \omega)$ for a simple atomic dilute gas with a realistic potential using the Boltzmann equation in order to check if it is really sensitive to the potential shape. We shall consider the (12-6) Lennard-Jones potential defined by $\phi(r)=4 \epsilon\left\{(\sigma / r)^{12}-(\sigma / r)^{6}\right\}$, where $\epsilon$ is the depth of potential and $\sigma$ is the distance at which $\phi(r)=0$. We focus only on the Lennard-Jones potential because this potential holds the main features of other realistic potentials, such as the steep repulsive short range interaction part with the long attractive tail. Instead of inspecting various potentials, we calculate $S(k, \omega)$ for the Lennard-Jones potential for various temperatures.

\section{THEORY}

The dynamic structure factor is defined as

$$
S(k, \omega)=\frac{1}{2 \pi} \int_{-\infty}^{\infty} d t e^{i \omega t} \sum_{i \neq j=1}^{N}\left\langle e^{i \mathbf{k} \cdot\left\{\mathbf{R}_{i}(0)-\mathbf{R}_{j}(t)\right\}}\right\rangle,
$$

where $k=|\mathbf{k}|, N$ is the total number of the atoms, $\mathbf{R}_{i}(t)$ is the position of the $i$ th atom at a time $t$, and $\langle\cdots\rangle$ is the average over an equilibrium ensemble. In the low density limit, this can be expressed using the Boltzmann operator as [6]

$$
S(k, \omega)=\frac{1}{\pi} \operatorname{Re}\left(\frac{1}{i \omega+i \mathbf{k} \cdot \mathbf{p} / m+n \Lambda_{B}}\right)_{1},
$$

where $n$ is the number density of atoms and $\Lambda_{B}$ is the Boltzmann operator. $\langle\cdots\rangle_{1} \equiv \int d \mathbf{p} f(p) \cdots$, where $f(p)$ is the Maxwellian distribution function, is the average over one particle ensemble. If either $\omega$ or $\mathbf{k} \cdot \mathbf{p} / m$ is much larger than $n \Lambda_{B}$, we can expand the right-hand side of Eq. (2) in terms of the density. This condition is translated as $\omega \tau \gg 1$ or $k l \gg 1$, where $l=1 / \sqrt{2} \pi n \sigma^{2}$ and $\tau=l \sqrt{\pi m / 8 k_{B} T}$ are the mean free path and the mean free time for the atom of a mass 
$m$, respectively. Here $k_{B}$ is the Boltzmann constant and $T$ is the absolute temperature. Up to the linear order in $1 / k l$, Eq.

(2) can be expanded as

$$
\begin{aligned}
\frac{S(k, \omega)}{S(k)} & =S^{(0)}(k, \omega)+n S^{(1)}(k, \omega)+O\left(n^{2}\right) \\
& =\frac{2}{\pi} \frac{\tau}{k l}\left\{e^{-4 \omega^{* 2} / \pi}+\frac{s_{11}(k, \omega)}{k l}+O\left((k l)^{-2}\right)\right\},
\end{aligned}
$$

where $\omega^{*}=\omega \tau / k l$ is a reduced frequency. The first term on the right-hand side represents the ideal gas term, which describes the free motion of atoms without collisions with each other. The second term, $s_{11}(k, \omega)$, is the first correction term in the expansion and is given by

$$
\begin{aligned}
s_{11}(k, \omega)= & \sqrt{\frac{k_{B} T}{\pi^{3} m}\left(\frac{k}{\sigma}\right)^{2}} \\
& \times \operatorname{Re}\left(\frac{1}{i \omega+i \mathbf{k} \cdot \mathbf{p} / m} \Lambda_{B} \frac{1}{i \omega+i \mathbf{k} \cdot \mathbf{p} / m}\right)_{1} \\
= & \frac{1}{8 \sigma^{2}} \sqrt{\frac{m}{\pi k_{B} T}} \\
& \times \operatorname{Re}\left\langle\frac{1}{i \omega^{*}+i \alpha \hat{\mathbf{k}} \cdot \mathbf{p}} \Lambda_{B} \frac{1}{i \omega^{*}+i \alpha \hat{\mathbf{k}} \cdot \mathbf{p}}\right)_{1} \\
\equiv & s_{11}\left(\omega^{*}\right),
\end{aligned}
$$

where $\hat{\mathbf{k}}=\mathbf{k} /|\mathbf{k}|$ and $\alpha=\sqrt{\pi / 8 m k_{B} T}$. Note that, for arbitrary potentials, the linear term in the expansion is scaled by a single parameter, $\omega^{*}$, and, therefore, independent of the wave vector. The Boltzmann operator is defined, for an arbitrary function of the momentum, $A(\mathbf{p})$, as [7]

$$
\begin{aligned}
\Lambda_{B} A= & \frac{4 \pi n}{m} \int d \mathbf{p}_{2} \int_{0}^{\infty} b d b p_{12} f\left(p_{2}\right)\left\{A\left(\mathbf{p}_{1 \infty}\right)+A\left(\mathbf{p}_{2 \infty}\right)\right. \\
& \left.-A\left(\mathbf{p}_{1}\right)-A\left(\mathbf{p}_{2}\right)\right\},
\end{aligned}
$$

where $b$ is the impact parameter of the collision of a pair of particles " 1 "' and ' 2 ,' $\mathbf{p}_{12}=\mathbf{p}_{1}-\mathbf{p}_{2}$ is a relative momentum of the pair. $\mathbf{p}_{1 \infty}$ and $\mathbf{p}_{2 \infty}$ are the momenta of the particles after the collision and are expressed as

$$
\left\{\begin{array}{l}
\mathbf{p}_{1 \infty}=\frac{\mathbf{p}_{1}+\mathbf{p}_{2}}{2}+\mathbf{p}_{12 \infty} \\
\mathbf{p}_{2 \infty}=\frac{\mathbf{p}_{1}+\mathbf{p}_{2}}{2}-\mathbf{p}_{12 \infty} .
\end{array}\right.
$$

The first term on the right-hand side of these equations represents the momentum of the center of mass. The second term is the relative momentum after the collision given by $\mathbf{p}_{12 \infty}=p_{12}\left\{\hat{\mathbf{e}}_{1} \cos \chi\left(p_{12}, b\right)+\hat{\mathbf{e}}_{2} \sin \chi\left(p_{12}, b\right)\right\}$, where $\hat{\mathbf{e}}_{1}$ and $\hat{\mathbf{e}}_{2}$ are the unit vectors parallel and perpendicular to $\mathbf{p}_{12}$, respectively, and $\chi(p, b)$ is the deflection angle given, for a continuous potential, by $[1,7]$

$$
\chi(p, b)=\pi-2 b \int_{r_{0}}^{\infty} d r \frac{1}{r^{2} \sqrt{1-m \phi(r) / p^{2}-b^{2} / r^{2}}},
$$

where $r_{0}$ is the zero of $1-m \phi(r) / p^{2}-b^{2} / r^{2}=0$.

$s_{11}\left(\omega^{*}\right)$ can be evaluated from Eqs. (4) and (5). After some straightforward mathematical manipulations, we obtain

$$
s_{11}\left(\omega^{*}\right)=\int_{0}^{\infty} d t F_{11}(t) \cos \omega^{*} t
$$

with

$$
\begin{aligned}
F_{11}(t)= & -\frac{128}{\pi^{3} \sigma^{2}} t e^{-\pi t^{2} / 32} \int_{0}^{\infty} d p \int_{0}^{\pi} \sin \theta d \theta \\
& \times \int_{0}^{\pi} d \phi \int_{0}^{\infty} d b b p^{3} e^{-8 p^{2} / \pi}\{\psi(\hat{\mathbf{k}} \cdot \mathbf{p} t, \hat{\mathbf{k}} \cdot \mathbf{p} t) \\
& +\psi(\hat{\mathbf{k}} \cdot \mathbf{p} t,-\hat{\mathbf{k}} \cdot \mathbf{p} t)-\psi\left(\hat{\mathbf{k}} \cdot \mathbf{p} t, \hat{\mathbf{k}} \cdot \mathbf{p}_{\infty} t\right) \\
& \left.-\psi\left(\hat{\mathbf{k}} \cdot \mathbf{p} t,-\hat{\mathbf{k}} \cdot \mathbf{p}_{\infty} t\right)\right\}
\end{aligned}
$$

where $\quad \hat{\mathbf{k}} \cdot \mathbf{p}=p \cos \theta, \quad \hat{\mathbf{k}} \cdot \mathbf{p}_{\infty}=p\{\cos \theta \cos \chi(p, b)$ $+\sin \theta \cos \phi \sin \chi(p, b)\}$, and $\psi(x, y) \equiv(\sin x-\sin y) /(x-y)$. $\theta$ and $\phi$ are the polar angles between $\hat{\mathbf{k}}$ and $\mathbf{p}$. Note that in Eq. (9), p stands for $\alpha \mathbf{p}$. We have evaluated the fourdimensional integration of Eq. (9) numerically using the Gaussian quadrature routine. After evaluating $F_{11}(t)$, the fast Fourier transform (FFT) routine was used to transfer to $s_{11}\left(\omega^{*}\right)$. We remark that this expression reduces to the hardsphere result [6] by inserting the expression for the deflection angle of the hard sphere [7]

$$
\cos \chi(p, b)=1-2\left(1-\frac{b^{2}}{\sigma^{2}}\right) \theta(\sigma-b),
$$

where $\theta(x)=1$ for $x \geqslant 0$ and $\theta(x)=0$ for $x<0$, is the Heaviside function.

\section{RESULTS}

In Fig. 1, we show $s_{11}\left(\omega^{*}\right)$ for the Lennard-Jones fluid with the argon parameter, $\epsilon / k_{B}=132 \mathrm{~K}$, at the temperature $T / T_{c}=2.0$, where $T_{c}=151 \mathrm{~K}$ is the critical temperature of argon. The experimental result by Verkerk et al. [5] for $k$ $=1.2 \mathrm{~nm}^{-1}$ at the same temperature and the hard-sphere result by Kamgar-Parsi et al. [6] are also shown. It appeared that the amplitude of the oscillatory curve for the LennardJones fluid is slightly larger but the qualitative behavior is the same as the hard-sphere result. The agreement with the experimental result is again rather poor, even qualitatively. In order to check how sensitive the result is to the depth of the potential, we have calculated $s_{11}\left(\omega^{*}\right)$ for several temperatures. In Fig. 2, we have plotted the results for the tem- 


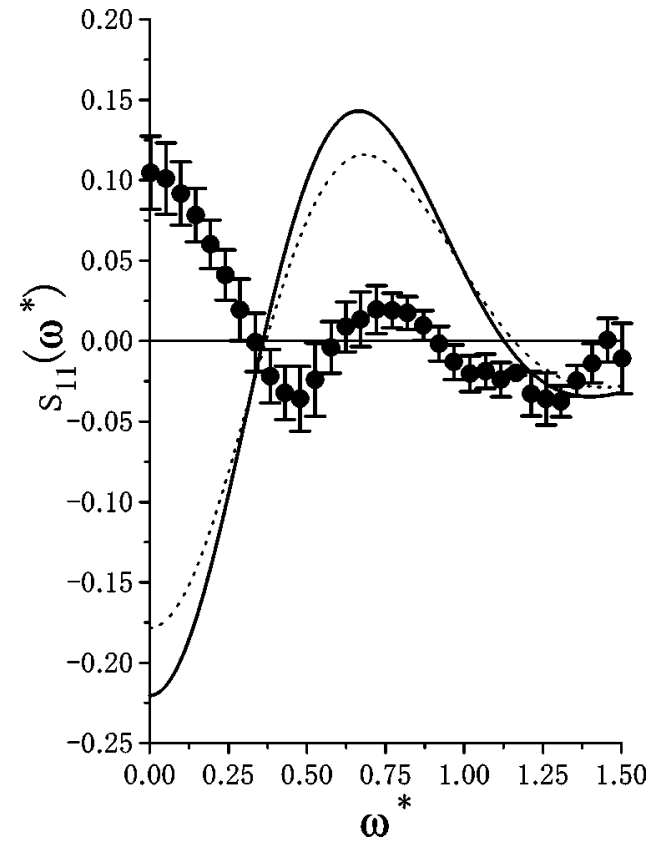

FIG. 1. $s_{11}\left(\omega^{*}\right)$ calculated for the Lennard-Jones potential (solid line), and the hard-sphere (dotted line) [6]. The experimental data by Verkerk et al. [5] for $k=1.2 \mathrm{~nm}^{-1}$ at $T / T_{c}=2.0$ is shown by closed circles.

peratures $T^{*}=1.0,2.0$, and 4.0 , where $T^{*}=k_{B} T / \epsilon$ is the reduced temperature. The hard-sphere result which is temperature-independent is also shown. Note that $T^{*}=1.0$ is below the corresponding critical temperature of argon $\left(T_{c}\right.$ $=151 \mathrm{~K})$. As one expects, the results get closer to the hardsphere result as temperature increases, where the attractive

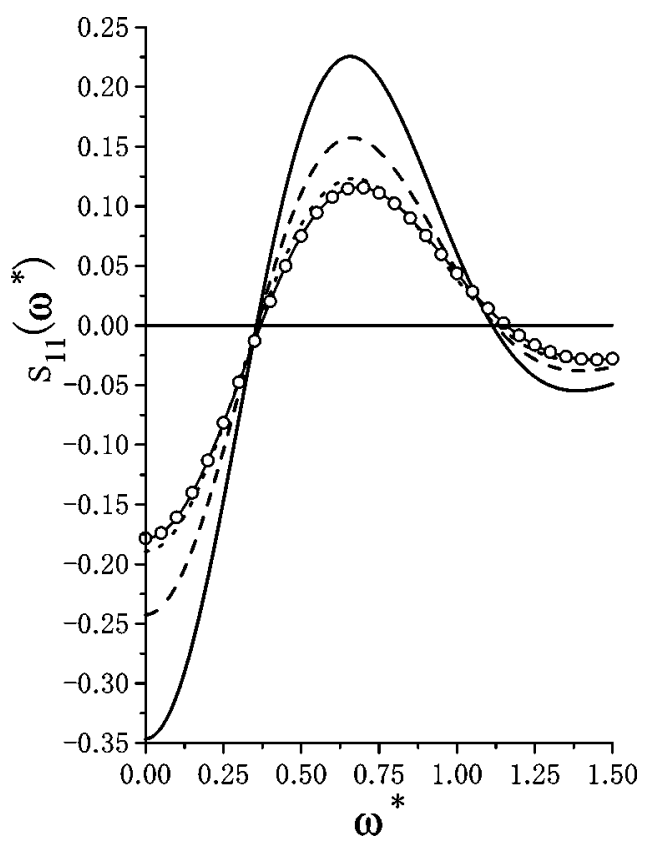

FIG. 2. $s_{11}\left(\omega^{*}\right)$ for the Lennard-Jones fluid for various temperatures: $T^{*}=1.0$ (solid line), $T^{*}=2.0$ (dashed line), $T^{*}=4.0$ (dotted line). The hard-sphere result is also shown (solid line with open circles). part of the potential is expected to influence the dynamics less. Even for low temperatures, we see that the qualitative behavior remains the same as that of the hard-sphere. Though the real potential for the dilute argon is slightly different from the Lennard-Jones potential, it is difficult to imagine that the minor difference of the potential shape or depth causes major qualitative differences in the results.

Another rationale which Verkerk et al. claimed to explain the strong wave vector dependence of $s_{11}\left(\omega^{*}\right)$ is that either $k l$ or $\omega \tau$, both of which are proportional to the density, were not big enough to make the density expansion valid. Recently, however, their group conducted similar experiments [8] but for samples with lower densities, down to less than $1.0 \mathrm{~nm}^{-3}$, whereas their experiments shown in Fig. 1 was for $n \leqslant 2.0 \mathrm{~nm}^{-3}$. In spite of the lower densities (larger $k l$ ), their results remained qualitatively the same. This implies that the infringement of the criteria of the density expansion was not the major reason for the discrepancies.

From these considerations, we may conclude that the potential shape does not affect the qualitative behavior of $s_{11}\left(\omega^{*}\right)$ as long as one uses the Boltzmann equation. Therefore, the discrepancies should be attributed to the validity of the Boltzmann equation itself on the length and time scales involved in the experiments. This conclusion is consistent with the results of molecular dynamics simulations by Bafile et al. [9]. They simulated the Lennard-Jones fluids with the parameters normally used for argon and calculated the leading term of the dynamic structure factor. Though their simulation results are preliminary and did not reproduce the experimental results perfectly, they still have a strong wave vector dependence and qualitatively the same behavior as the experimental results.

\section{DISCUSSION}

Here we propose several possibilities to explain discrepancies between theories and experiments. In order to use the Boltzmann equation for the system concerned here, there are several prerequisite conditions. First, the length scale observed should be larger than that of the intermolecular interaction. For the Lennard-Jones or hard-sphere potential, the length of the interaction is the order of $\sigma$. Therefore, in order to apply the Boltzmann equation approach, $k \sigma$ must be sufficiently smaller than 1 . Second, the time scale should be large enough compared with the collision time. The collision time is the order of $\tau_{c}=\sigma \sqrt{m / k_{B} T}$ and $\omega \tau_{c} \ll 1$ should be satisfied. Combining both criteria for the density expansion in the dynamic structure factor to hold, we conclude that $1 / l \ll k \ll 1 / \sigma$ and $1 / \tau \ll \omega \ll 1 / \tau_{c}$ should be satisfied. Using the Lennard-Jones diameter for argon $\sigma=0.34 \mathrm{~nm}$, we see that the wave vector range in Verkerk's experiment was 0.1 $\leqslant k \sigma \leqslant 0.51$. This might not be small enough to employ the Boltzmann equation. On the other hand, the latter condition is translated, in the dimensionless form, to $1 / k l \ll \omega^{*}$ $\ll 1 / k \sigma$. Since the window of the frequency in the experiment is $0 \leqslant \omega^{*} \leqslant 1.5$, the upper limit of the condition seems to be barely satisfied.

If $\omega$ is not small enough, one needs to generalize the Boltzmann operator $\Lambda_{B}$ to the time-dependent memory ker- 
nel, $\int_{0}^{\infty} d t^{\prime} \Lambda_{B}\left(t-t^{\prime}\right)$, to include the detailed scattering process. If $k$ is not small enough, one needs to extend $\Lambda_{B}$ to the wave-vector-dependent one $\Lambda_{B \mathbf{k}}$. The Boltzmann operator has been generalized to the finite wave vector region under the scheme of the Enskog theory $[6,10]$, but the Enskog theory can be applied only for the hard-sphere system. A similar scheme for arbitrary potentials seems necessary. Recently, Miyazaki et al. have developed the "Enskog theory", for the continuous potential in order to study several properties of the self-diffusion of a tagged particle in the LennardJones potential [11]. Their theory made it possible to calculate the time-dependent memory kernel for fluids with arbitrary potentials, which provides the information of the dynamical processes of the binary collisions directly. They found that the memory kernel for the friction coefficient decays with a finite time and is sensitive to the potential shape, contrary to the instant $\delta$-function-like decay for the hardsphere fluid. Their theory, however, is restricted to the small wave vector region. Generalization of the theory to the finite wave vector region seems possible and may provide the clue to understand the current problem. It is well-known that the static properties of dilute gases are very sensitive to the shape of the intermolecular potential. For example, the static structure factor $S(k)$ for the Lennard-Jones potential is quite different from that for the hard-sphere at finite wave vectors. One would expect that this is also the case for the dynamic functions such as $S(k, \omega)$. Developing the wave-vectordependent "Enskog theory" for arbitrary potentials is indispensable to resolve the puzzling discrepancies between theory and experiments. A study in this direction is under way.

\section{ACKNOWLEDGMENTS}

We are grateful to Dr. P. Verkerk for providing us with his experimental data and fruitful discussions. We thank Professor Biman Bagchi for encouragement and enlightening discussions. The work is supported in part by grants from the Japan Society for the Promotion of Science (JSPS).
[1] D. A. McQuarrie, Statistical Mechanics (Harper Collins, New York, 1973).

[2] A. Teitsma and P. A. Egelstaff, Phys. Rev. A 21, 367 (1980).

[3] F. Barocchi, M. Zoppi, and P. A. Egelstaff, Phys. Rev. A 31, 2732 (1985).

[4] H. Fredrikze, J. B. van Tricht, A. A. van Well, R. Magli, P. Chieux, and F. Barocchi, Phys. Rev. Lett. 62, 2612 (1989).

[5] P. Verkerk, U. Bafile, F. Barocchi, L. A. de Graaf, J.-B. Suck, and H. Mutka, Phys. Rev. Lett. 67, 1262 (1991).

[6] B. Kamgar-Parsi, E. G. D. Cohen, and I. M. de Schepper, Phys. Rev. A 35, 4781 (1987).
[7] P. Résibois and M. de Leener, Classical Kinetic Theory of Fluids (Wiley, New York, 1977).

[8] B. Mos, Ph.D. thesis, Delft University of Technology, 2000 (unpublished).

[9] U. Bafile, F. Barocchi, and M. Neumann, J. Mol. Struct. 296, 221 (1993).

[10] I. M. de Schepper and E. G. D. Cohen, J. Stat. Phys. 27, 223 (1982).

[11] K. Miyazaki, G. Srinivas, and B. Bagchi, J. Chem. Phys. 114, 6276 (2001). 